\title{
ESTIMATIVA DE EMISSÃO DE MONÓXIDO DE CARBONO E DE METANO PARA O NORDESTE DO BRASIL
}

\author{
Ronabson C. Fernandes ${ }^{1}$, Judith J. Hoelzemann ${ }^{2}$ \\ Programa de Pós-Graduação em Ciências Climáticas -UFRN \\ ${ }^{1}$ ronabson@ hotmail.com \\ ${ }^{2}$ judith.hoelzemann@gmail.com
}

\section{SUMMARY}

In this study carbon monoxide and methane emissions from agricultural cane sugar production provide were estimed by IBGE. It was found that states Alagoas and Pernambuco were the largest emitters of methane and carbon monoxide into the atmosphere.

Keywords: methane, carbon monoxide, sugar cane.

\section{INTRODUÇÃO}

O entendimento das emissões provenientes da queima dos resíduos agrícolas é de grande importância para a modelagem química atmosférica, economia, saúde, e para a compreensão do comportamento das espécies químicas emitidas na atmosfera, que tem um efeito no meio ambiente e no clima. Segundo o IPCC (2007) o metano $\left(\mathrm{CH}_{4}\right)$ é cerca 21 vezes mais eficiente na retenção de calor do que o $\mathrm{CO}_{2}$.

O objetivo desse trabalho foi estimar as emissões de monóxido de carbono e de metano a partir da produção de cana-de-açúcar para o Nordeste Brasileiro (NEB), para contribuir para a quantificação de emissões da região.

\section{MATERIAIS E MÉTODOS}

Para a realização desse estudo, utilizaram-se dados municipais de produção da canade-açúcar provenientes do Instituto Brasileiro de Geografia e Estatística (IBGE, 2013), dos 
estados de Alagoas (AL), Bahia(BA), Ceará(CE), Maranhão(MA), Paraíba (PB), Pernambuco (PE),Piauí(PI), Rio Grande do Norte (RN) e Sergipe (SE), no período de 2007 a 2011, num total de 1.794 municípios estudados. Ressalta-se que nem todos os municípios examinados foram produtores de cana-de-açúcar. Esses dados foram organizados por estado e ano e, posteriormente, serviram para estimar as emissões de $\mathrm{CH}_{4}$ e de $\mathrm{CO}$ a partir da eq.1 (Lima et al. ,2010).

$$
M c=\left(P \times R \times R_{S} \times R_{e} \times R_{q}\right) \times T c
$$

Sabendo que $\mathrm{M}_{\mathrm{C}}=$ massa total do carbono ou nitrogênio; $\mathrm{P}$ = produção da cana-de-açúcar, em gigagramas $\left(\mathrm{Gg}=10^{9}\right.$ gramas $) ; \mathrm{R}=$ relação resíduo produção; $\mathrm{R}_{\mathrm{s}}=$ conteúdo de matéria seca do resíduo; $\mathrm{R}_{\mathrm{e}}=$ proporção de resíduos expostos ã queima; $\mathrm{R}_{\mathrm{q}}=$ fração oxidada de resíduos durante a queima e $\mathrm{T}_{\mathrm{c}}=$ conteúdo de carbono nos resíduos. Foi considerada a queima de $100 \%$ desses resíduos e, um fator de 0,0028307 ( $\left.\mathrm{CH}_{4}\right)$ e de 0,0594440 (CO).

\section{RESULTADOS E DISCUSSÕES}

Na Figura 1, é mostrada a emissão anual de $\mathrm{CH}_{4}$ pela queima dos resíduos da canade-açúcar para a atmosfera. Observou-se que a maior emissão de $\mathrm{CH}_{4}$ no $\mathrm{NEB}$ ocorreu em 2011, com 28,61 Gg, seguidos de 28,38 Gg (em 2008) e 26,81 Gg (em 2009). Em média, os estados de AL e PE foram os maiores emissores com 10,30Gg/ano e 7,53Gg/ano, respectivamente. Ainda, o máximo valor obtido para Alagoas ocorreu em 2011, com 11,19Gg, e em 2008 para PE, 7,79Gg. Contudo, em média, Piauí (0,31 Gg/ano) e Ceará $(0,86 \mathrm{Gg} / \mathrm{ano})$ tiveram os menores valores estimados. Ainda, o menor valor estimado ocorreu em PI, com 0,29Gg/2008, e CE, 0,86Gg/2007. 


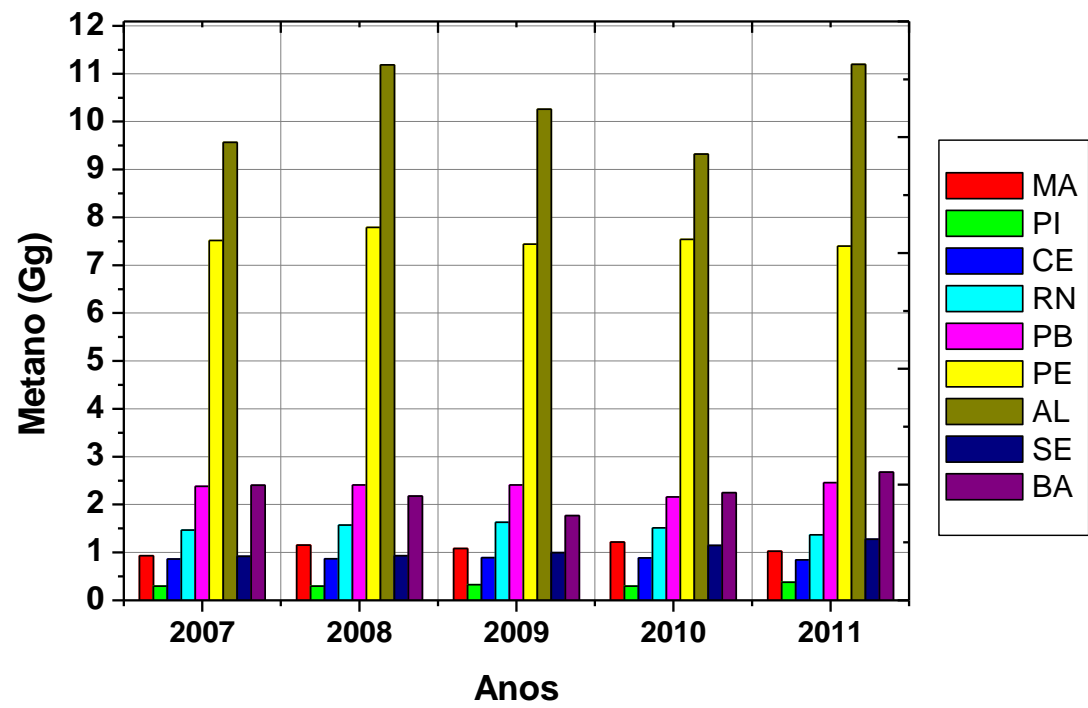

Figura 1 - Emissão anual de metano, em Gg, por estado, de 2007 a 2011.

Em relação às emissões anuais de CO no NEB, 2011, 2008 e 2007, obtiveram os máximos valores em 600,96 Gg, 595,93Gg e 553,22Gg, respectivamente. Especificamente, em 2011, os estados no NEB que mais emitiram CO para a atmosfera foram AL $(235,11$ Gg/ano), PE (155,35 Gg/ano) e PB (51,57 Gg/ano), respectivamente (Figura 2)

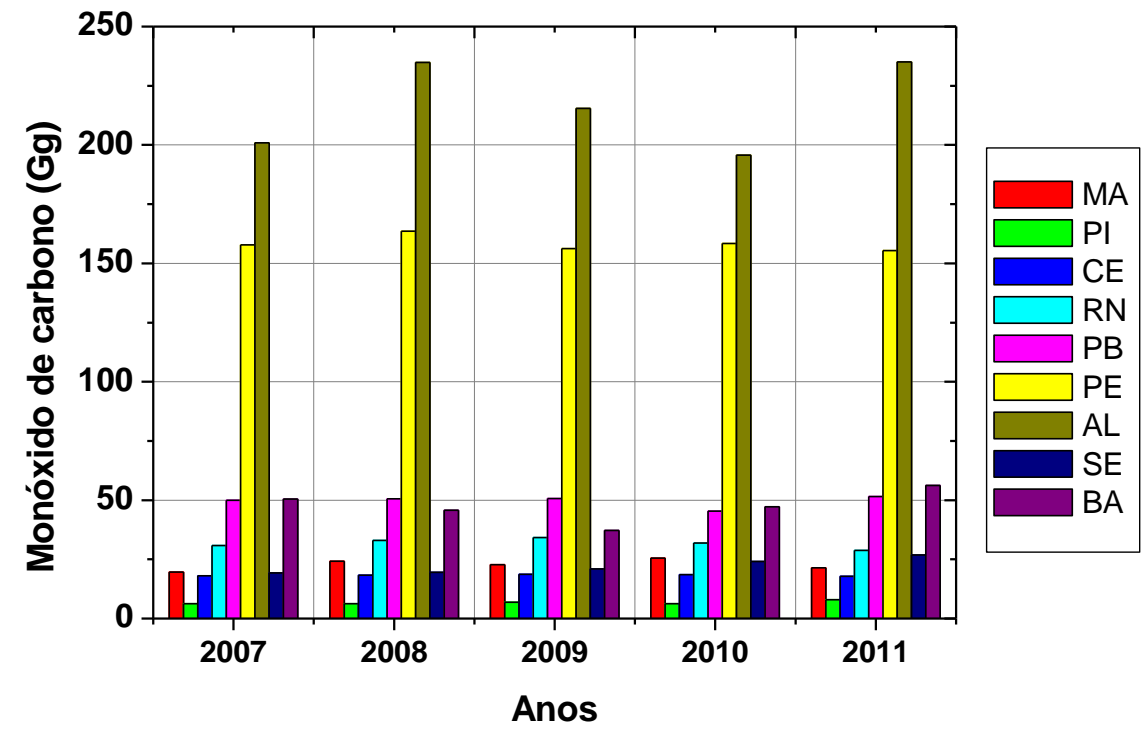

Figura 2 - Emissão anual de monóxido carbono, em Gg, por estado, de 2007 a 2011. 


\section{CONCLUSÕES}

Diante dos resultados obtidos a partir das estimativas de da queima da cana-deaçúcar, foi mostrado que os estados de Alagoas e Pernambuco foram os maiores emissores de metano e de monóxido de carbono para atmosfera.

\section{AGRADECIMENTOS}

À FAPERN/CAPES, pela concessão da bolsa de Doutorado.

\section{BIBLIOGRAFIA}

IBGE, Instituto Brasileiro de Geografia e Estatística. Produção Agrícola Municipal, disponível em: http://www.sidra.ibge.gov.br/bda/tabela/listabl.asp?c=1612\&z=t\&o=11 Acessado em 03 Maio de 2013.

INTERGOVERNMENTAL PANEL ON CLIMATE CHANGE (IPCC). Climate Change 2007: synthesis report. PACHAURI, R. K.; REISINGER, A. (Ed.). Contribution of Working Groups I, II and III to the Fourth Assessment Report of the Intergovernmental Panel on Climate Change. Geneva, Switzerland, 2007.

LIMA, M. A.; LIGO, M. A. V.; PESSOA, M. C. P. Y.; NEVES, M. C.; CARVALHO, E. C. de Emissões de gases de efeito estufa na queima de resíduos agrícolas. Brasília: Ministério da Ciência e Tecnologia: Embrapa, 2010. Segundo Inventário Brasileiro de Emissões e Remoções Antrópicas de Gases de Efeito Estufa - Relatórios de referência. 